\title{
SHEAR BOND STRENGTH OF ORTHODONTIC BRACKETS BONDED WITH TOTAL ETCH ADHESIVE SYSTEMS CONTAINING SILVER NANOPARTICLES “AN IN VITRO STUDY”
}

\author{
Kareem Hamdy*, Mona I. Riad**, Amir Hafez ${ }^{* * * *}$ and Amany S. Fekry****
}

\begin{abstract}
Objective: This study was conducted to assess the shear bond strength of orthodontic brackets bonded with total etch adhesive systems containing silver nanoparticles (AgNPs) and interfacial analysis using Environmental Scanning Electron Microscope (ESEM).

Materials and methods: The AgNPs was synthesized and characterized by (UV-Vis) spectroscopy and Transmission Electron Microscope (TEM). Adper Single Bond 2 was used as the total etch $(\boldsymbol{T E})$ adhesive. 48 teeth were divided into 40 teeth for shear bond strength test and 8 teeth for ultra- morphologic examination. For shear bond strength test 40 teeth were randomly divided into two equal groups according to the addition of silver nanoparticles, $(\boldsymbol{T E})$ teeth were bonded with adhesive not containing silver nanoparticles and (TES) teeth were bonded with adhesive containing silver nanoparticles. Each group was subdivided into 2 subgroups: $\boldsymbol{C}_{\boldsymbol{I}}$ (10 specimens): 24 hours aging period. $\boldsymbol{C}_{2}$ (10 specimens): 3 months aging period. Finally two representative samples of each group were analyzed using an (ESEM) for interfacial analysis.
\end{abstract}

Result: After 24 hours aging, there was non-significant difference between mean shear bond strength values of the adhesive contain silver nanoparticles $(\mathbf{5 . 0 9 \pm 1 . 6 9 )}$ and the nanoparticles free adhesive (4.69 $\pm \mathbf{1 . 4 2})$. Also after 3 months aging there was non-significant difference between both adhesives with $(\mathbf{6 . 3 1} \pm \mathbf{1 . 1 2})$ and without $(\mathbf{5 . 1 4} \pm \mathbf{1 . 1 6})$ addition of silver nanoparticles.

Conclusions: The addition silver nanoparticles to total-etch adhesive systems did not hinder bonding of resin composite to enamel. Silver nanoparticles didn't antagonize penetration of resin tags into enamel. Bonding of brackets with resin composite using total etch adhesive system containing and non- containing Silver nanoparticles doesn't deteriorate with time.

\footnotetext{
* MSC in Operative Dentistry, Cairo University

** Professor of Operative Dentistry, Faculty of Oral and Dental Medicine, Cairo University

*** Lecturer of Operative Dentistry, Faculty of Oral and Dental Medicine, Cairo University.

**** Researcher of Orthodontic Dentistry , Research Institute of Ophthalmology
} 


\section{INTRODUCTION}

During treatment with fixed orthodontic appliances certain problems may occur, such as decalcification of the enamel around the brackets, which is considered the most common problem in patients undergoing orthodontic treatment with fixed appliances Attar $N \mathbf{2 0 0 7}{ }^{(I)}$. So several attempts have been made in this field to incorporate antibacterial agents in resin composite as fluorides and chlorhexidine (Scougall Vilchis $R J$ 2007) ${ }^{(2)}$. However, besides their questionable biological effect they always resulted in inferior mechanical properties. With the emergence of nanotechnology and nanoparticles as hydroxyapatite and Nano silver. Attempts have been made to incorporate Nano silver particles as shown to inhibit bacterial growth around bracket margins (Polat et al., 2005) $)^{(3)}$.

Silver has a long history of use in medicine as an antimicrobial agent as reported by Arokiyaraj et al., 2014(4). Resin composites containing silver ion-implanted fillers that release silver ions have been found to have antibacterial effects on oral streptococci. In the literature there are no reports of studies that have analyzed the strength of the bracket/tooth bond after aging to verify its longevity and integrity, so it seems value to study the effect of incorporating silver nanoparticles in (TE) adhesive systems on the shear bond strength of orthodontic brackets, assess the bond durability after aging and examination the interface using ESEM with null hypothesis: Addition of silver nanoparticles to dental adhesives used to bond orthodontic brackets will not affect shear bond strength.

\section{MATERIALS AND METHODS}

The materials used in this study were filtek Z350 XT universal resin composite restorative (3M ESPE, St. Paul MN, USA), (TE) Adhesive Adper Single Bond 2 (3M ESPE, St. Paul MN, USA) as the control and total etch with silver nanoparticles (TES).

\section{AgNPs synthesis and characterization}

The AgNPs was synthesized using ethanol as a reducing agent, where $10 \mathrm{ml}$ of $1 \%(\mathrm{w} / \mathrm{v})$ ethanol solution of PVP and $0.1 \mathrm{~m} 1$ of $0.1 \mathrm{M}$ silver nitrate were taken in a $25 \mathrm{ml}$ closed conical flask and placed in a microwave oven (model KOG-IA4H) that was operated at the $100 \%$ power of $800 \mathrm{~W}$ and frequency $2450 \mathrm{MHz}$ for 5 seconds. Pal et al, 2009 ${ }^{(5)}$.

The produced silver nanoparticles were characterized using Ultraviolet-visible absorption (UV-Vis) spectroscopy (Cary 5000 UV- Vis -NIR Spectrophotometer, GMI, USA) and Transmission electron microscope (TEM) (JEOL JEM-2011 (JEOL Ltd, Japan).

\section{Incorporation of AgNPs}

Ethanol solution containing $0.5 \mu \mathrm{g} / \mathrm{ml}$ silver nanoparticles were added to the adhesive and mixing was. This resulted in modified adhesive systems containing $0.5 \mu \mathrm{g} / \mathrm{ml}$ silver nanoparticles (Magalhaes et al., 2012) ${ }^{(6)}$ which is the least concentration that has highest antibacterial effect.

\section{Selection of teeth:}

48 freshly extracted human upper central incisors were collected from The National Institute for Diabetes. The teeth were cleaned, polished with pumice and rotating brush and inspected under magnifying lens $(\times 7)$ for intact labial enamel surface free of caries, hypoplasia and no cracks. The teeth were stored in a glass jar containing $0.1 \%$ Thymol at $4 \mathrm{C}^{\circ}$ till usage and for no longer than one month.

\section{Preparation and Grouping of teeth}

Chemical cured acrylic resin (Acrostone chemical cure acrylic, 300640, Egypt) was mixed and poured inside Teflon mold then the root of each tooth was mounted in the mold up to the cementoenamel junction. Care was taken to assure that the long axis of each tooth was perpendicular on the mold edge using surveyor. 
The teeth were divided into two groups: 40 teeth for shear bond strength test (20 teeth for each group) and 8 teeth for ultra- morphologic examination (Two representative samples of each group). For shear bond strength test the teeth were randomly divided into two equal groups of 20 teeth each according to the addition of silver nanoparticles $(\boldsymbol{T E})$ teeth were bonded with adhesive not containing silver nanoparticles and (TES) teeth were bonded with adhesive containing silver nanoparticles. Each group was divided into 2 subgroups: $\boldsymbol{C}_{\boldsymbol{1}}$ (10 teeth): 24 hours aging period. $\boldsymbol{C}_{2}$ (10 teeth): 3 months aging period.

\section{Brackets bonding}

TE \& TES: Etching was performed at bracket place in the middle of the middle third of the labial surface using Scotchbond etchant (37\% phosphoric acid by weight) for 15 seconds then rinsed thoroughly by air-water spray for 10 seconds. Excess water was dried by oil free air for 5 seconds leaving the surface almost wet. Immediately after drying 2-3 consecutive coats of adhesive was applied to etched enamel by disposable micro brush for 15 seconds with gentle agitation.

Photo activation was performed for 10 seconds using the light curing system Elipar S10 (Elipar S10 (3M ESPE, St Paul, MN, USA) with $1200 \mathrm{~mW} /$ $\mathrm{cm}^{2}$ intensity. After application of adhesive agent, "Filtek ${ }^{\mathrm{TM}}$ Z350 XT resin composite was used to hold bracket in place by placing the composite on the base of the bracket then placing the brackets using bracket holder on the middle of the middle third of the labial surface of the tooth and eliminate excess composite using dental probe. Photo activation was performed for 40 seconds at zero distance then stored in artificial saliva at $37^{\circ} \mathrm{C}$ in an incubator. Saliva was refreshed every 24 hours in the three months aging period group.

\section{Shear test procedures}

Each specimen with the bonded bracket was secured with tightening screws to the lower fixed compartment of the universal testing machine (Instron, S.A.S. CAT. NO. 2710-113, France). A loop prepared from an orthodontic wire $(0.14 \mathrm{~mm}$ in diameter) was wrapped around the bonded bracket assembly close to the base of the bracket (Braga et al., 2010) ${ }^{(7)}$. A shearing load with tensile mode of force was applied via the universal testing machine at a crosshead speed of $0.5 \mathrm{~mm} / \mathrm{min}$.

\section{Ultra morphologic examination by ESEM}

Two representative samples of each group were prepared for ultra- morphologic examination of tooth-restoration interface using ESEM after 24 hours and 3 months aging periods.

\section{RESULTS}

\section{AgNPs synthesis and characterization}

The Ultraviolet-visible absorption (UV-Vis) spectroscopy used to determine the characteristic optical properties of silver nanoparticles. The absorption curve showed that the peak was at $407 \mathrm{~nm}$ and the maximum absorption peak occurred at 24 hours because of the surface Plasmon resonance of silver nanoparticles indicating the production of spherical nanoparticles (fig.1). Transmission electron microscope (TEM) showed that the size of the silver nanoparticles was found to be in the range of 3-20 nm, and they had a smooth surface and polydispersed particles (fig.2).

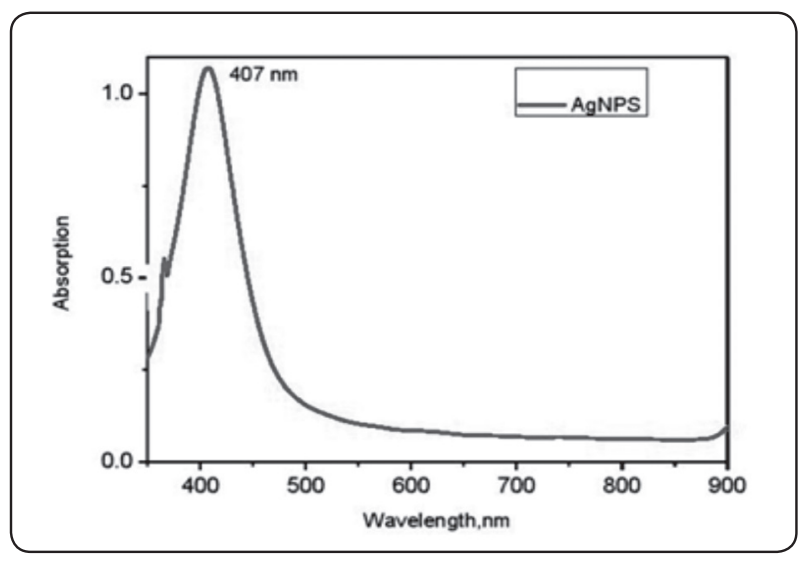

Fig. (1) The absorption rate curve of silver nanoparticles solution 


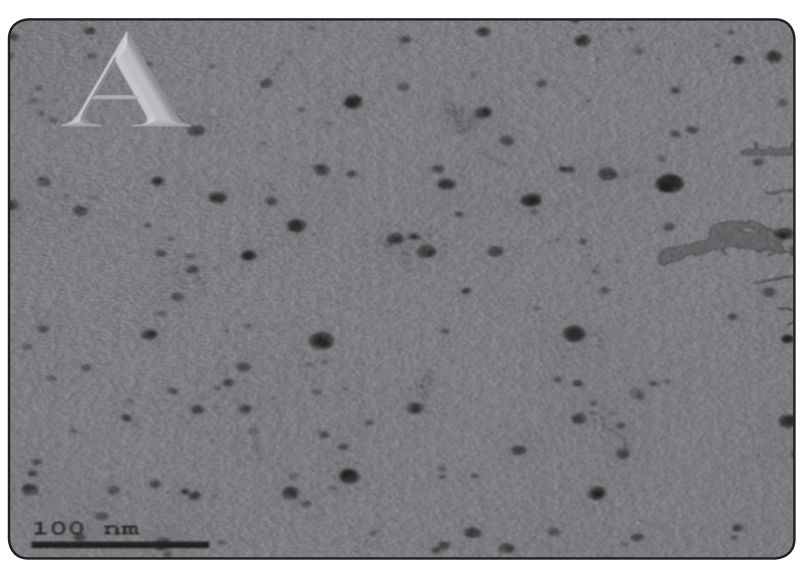

Fig. (2) TEM image of silver nanoparticles

After 24 hours aging period there was non significant difference in the shear bond strength between the adhesives with addition of silver nanoparticles and without silver nanoparticles, where mean and SD shear bond strength values of total etch adhesive with addition of silver nanoparticles was $\mathbf{( 5 . 0 9 \pm} \mathbf{1 . 6 9})$ and without

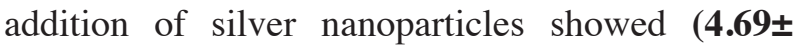
1.42). After 3 months aging there was also nonsignificant difference between both groups, where mean and SD shear bond strength values of totaletch adhesive with addition of silver nanoparticles was (6.31 \pm 1.12$)$ and without silver nanoparticles showed $(\mathbf{5 . 1 4} \pm \mathbf{1 . 1 6})$. The increase in the shear bond strength values of groups tested after 3 months was non significant from 24 hours.

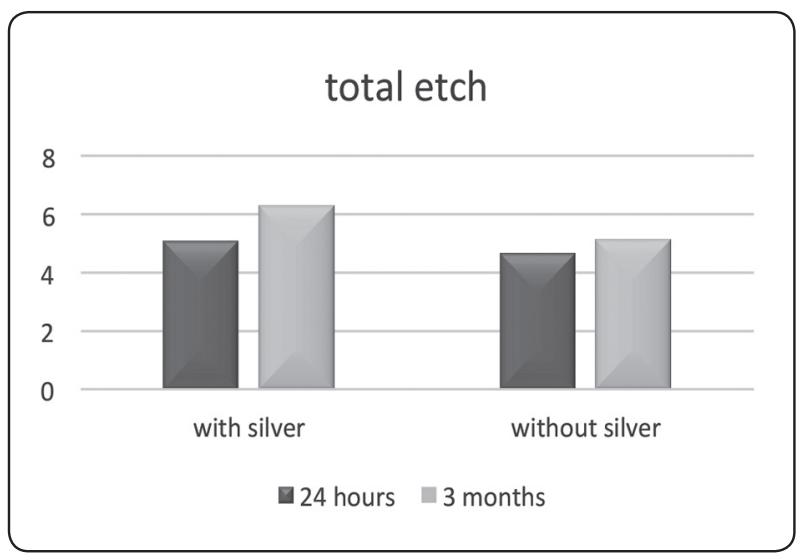

Fig. (3) Bar chart showing the mean Shear Bond Strength (MPa) for different storage periods.

\section{Ultra-morphologic examination by ESEM}

In both 24 hours and 3 months groups photomicrograph showed that tooth-resin composite interface has thin irregular hybrid layer and wellformed resin tags within the enamel with absence of any gap (fig. 4). Same appearance was observed for $\boldsymbol{T E S}$ group were photomicrograph showed that enamel-resin composite interface has thin irregular hybrid layer and well-formed resin tags within the enamel with absence of any gap and micro porosities; in addition to, presence of silver nanoparticles within and at the base of adhesive layer (fig. 5).

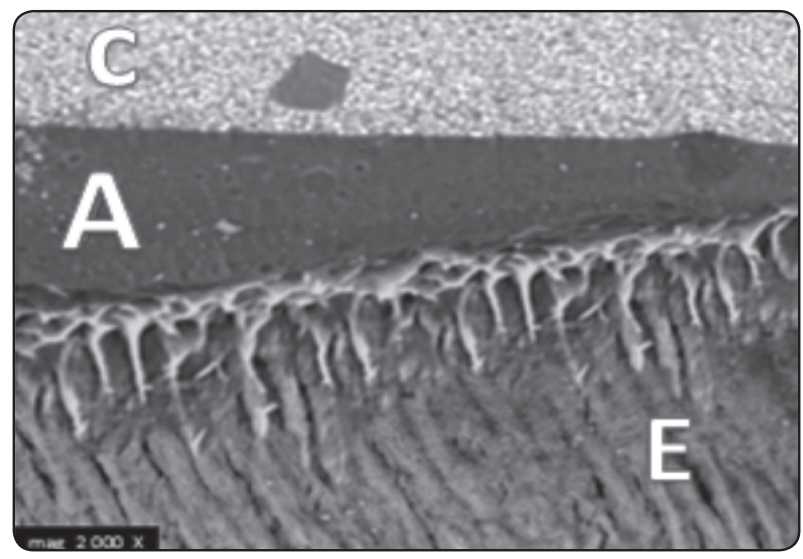

Fig. (4) Enamel-resin composite interface for Total etch after $24 \mathrm{hrs}$ aging period without silver C: composite, A: adhesive, E: enamel

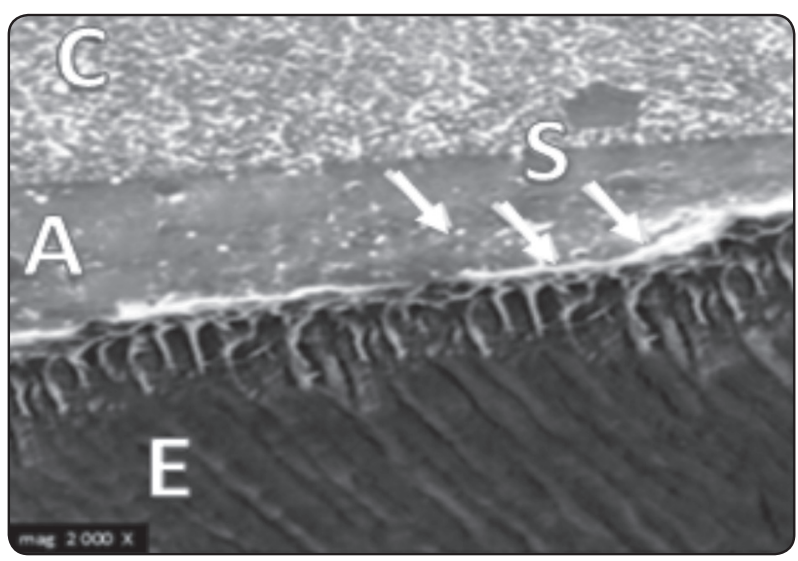

Fig. (5) E namel-resin composite interface for Total etch with silver nanoparticles after $24 \mathrm{hrs}$ aging period with silver $\mathrm{C}$ : composite, $\mathrm{S}$ : silver nanoparticles within and at the base of adhesive 


\section{DISCUSSION}

Several trials have attempted to reduce microbial adhesion to restorative materials, and the results are promising. These trials showed the development of resin-based restorative materials with antibacterial effect reached by addition of some agents as antibacterial monomer, chlorhexidine or titanium dioxide nanoparticles Bolzan et al., 2014 ${ }^{(8)}$.

Silver nanoparticles (AgNPs) have become a focus of interest because they play a significant role in biological systems, living organisms, and medicine Mie et al., 2013 ${ }^{(9)}$. Silver nanoparticles was used as they have significant antibacterial effect, The antimicrobial mechanism was suggested to be silver ions inactivating vital enzymes of bacteria, causing DNA to lose its replication ability, leading to cell death Cheng et al., 2012 ${ }^{(10)}$. Also cytotoxic assays revealed no toxicity of AgNPs toward mouse embryo fibroblast cells as concluded by Arokiyaraj et al., 2014(5), therefore, these particles were safe to use.

In addition, the size of 3-20 nm was used in this study because the smallest nanoparticles synthesized showed more antibacterial activity than large particles because small sized particles affect a large surface area of the bacteria Cheng et al., 2012 ${ }^{(10)}$. Also the concentration of silver used in this study was $0.5 \mu \mathrm{g} / \mathrm{ml}$ as Magalhaes et al., $2012^{(6)}$ showed that this concentration had better antibacterial activity with silver incorporation than commercial adhesives.

According to Chacko et al. $2013^{(11)}$ the major advantage of ESEM over normal scanning electron microscopes is that it is not necessary to make nonconductive samples conductive, and thus, their original characteristics can be preserved for further testing or manipulation.

In this study, it was found that there was no statistical significant difference in shear bond strength between 24 hours and 3 months aging period for both total etch adhesives either with or without silver nanoparticles denoting stable reliable bond when using total etch adhesive system in enamel. This was verified in both photomicrograph that showed well-formed resin tags with thin hybrid layer, this finding was supported by Foong et al., 2006 $^{(12)}$ who mentioned that pre-etching the intact enamel with $37 \%$ phosphoric acid resulted in deeper etch pattern, formation of longer resin tags and increased shear bond strength.

Foong et al., 2006 ${ }^{(12)}$ claimed that in etch and rinse adhesive system, the proper etching of enamel surface lead to adequate hybridization, resin tag formation with good sealing and adaptation which decrease rate of water sorption and preserve bond strength with time. the resin tags provides a threedimensional grasp of the etched enamel. This may prevent crack propagation via crack branching or deflection that consume fracture energy and preserve bond strength to enamel with time.

The addition of spherical shape silver nanoparticles with 3-20 nm size and $0.5 \mu \mathrm{g} / \mathrm{mL}$ concentration to total-etch adhesive systems did not hinder bonding of resin composite to enamel. Furthermore, the increase in shear bond strength with AgNPs may be attributed to that the nanofillers can improve adhesion at the interface between the restorative material and the tooth structure through increasing mechanical strength of the adhesive layer and providing structural reinforcement. In fact, the nanofillers are stress absorbing and have the role of an elastic layer between dental composite and enamel according to Akhavan et al., 2013 ${ }^{(13)}$. Furthermore orthodontic adhesive containing silver nanoparticles can contribute to preventing enamel demineralization around brackets without significant effects on the physical properties of adhesive. However increasing the size and concentration of AgNPs to adhesives may decrease shear bond strength due to an agglomeration of particles, creating defect points that act as stress raiser and lead to formation and crack propagation Frankenberger et al $2005^{(14)}$. As revealed in our 
photomicrograph that the AgNPs were present within and at the base of adhesive layer therefore AgNPs didn't antagonize penetration of resin tags into enamel and formation of hybrid layer. Hence the null hypothesis could be accepted.

\section{CONCLUSIONS}

\section{Under the limits of the present study it could be concluded that}

The addition silver nanoparticles to total-etch adhesive systems did not hinder bonding of resin composite to enamel. Silver nanoparticles didn't antagonize penetration of resin tags into enamel. Bonding of brackets with resin composite using total etch adhesive system containing and non- containing Silver nanoparticles doesn't deteriorate with time.

\section{REFERENCES}

1. Attar N, Taner TU, Tulumen E, Korkmaz Y. (2007). Shear bond strength oforthodontic brackets bonded using conventional vs one and two step self-etching/adhesive systems. Angle Orthodist;77:518-23

2. Scougall Vilchis J., Yamamoto S., Kitai N., Hotta M., Yamamoto K(2007). Shear bond strength of a new fluoride-releasing orthodontic adhesive. Dental materials Journal;26:45-51

3. Polat O, Uysal T \& Karaman AI. (2005). Effects of a chlorhexidine varnish on shear bond strength in indirect bonding. Angle Orthodontist; 75:1036-40.

4. Arokiyaraj S., Arasu V., Vincent S., Prakash U., Choi S. H. (2014). Rapid green synthesis of silver nanoparticles from Chrysanthemum indicum $\mathrm{L}$ and its antibacterial and cytotoxic effects: an in vitro study. International Journal of Nanomedicine, 9, 379-88.

5. Pal S, Tak Y., Joardar J., Kim W., Lee J., Han M, Song M (2009) Nanocrystalline silver supported on activated carbon matrix from hydrosol: antibacterial mechanism under prolonged incubation conditions. Journal of Nanoscience and Nanotechnology 9:2092-2103

6. Magalhaes R., Santos B., Lopes L., Estrela A., Torres É. M., Carrião M. (2012). Nanosilver Application in Dental Cements. Nanotechnology, 1-6.

7. Braga R.(2010), Adhesion to tooth structure: a critical review of "macro" test methods. Dental Materials 26, $38-49$.

8. Bolzan P., Augusto J., Agnelli M., Wesley C. \& Souza O. De. (2014). Addition of Silver Nanoparticles to Composite Resin : Effect on Physical and Bactericidal Properties In Vitro, Brazilian Dental Journal, 25(2): 141-145.

9. Mie R., Samsudin W., Din B., Ahmad A., Ibrahim N. \& Adnan N. A. (2013). Synthesis of silver nanoparticles using the lichen Parmotrema praesorediosum. International Journal of Nanomedicine, 9, 121-127.

10. Cheng L., Zhang K., Melo M. a. S., Weir M. D., Zhou X., \& Xu K. (2012). Anti-Biofilm Dentin Primer With Quaternary Ammonium And Silver Nanoparticles. Journal of Dental Research, 91, 598-604.

11. Chacko P., Kodoth J.(2015) Recycling stainless steel orthodontic brackets with Er:YAG laser - An Environmental Scanning Electron Microscope And Shear Bond Strength Study. Journal of Orthodontic Science,2(3)76-94

12. Foong K. Lee C. Nguyen G., Tang D., Austin C. Ch'ng(2006). Comparison of microshear bond strengths of four self-etching bonding systems to enamel using two test methods. Australian Dental Journal,51, 252-257

13. Akhavan A., Sodagar A., Mojtahedzadeh F. \& Sodagar K. (2013). Investigating the effect of incorporating nanosilver/nanohydroxyapatite particles on the shear bond strength of orthodontic adhesives. Acta Odontologica Scandinavica, 71, 1038-42.

14. Frankenberger R. \& Tay F. (2015). Self-etch vs etchand-rinse adhesives: effectof thermo-mechanical fatigue loading on marginal quality of bonded resincomposite restorations. Dental Materials, 21, 397-412. 